\title{
A THREE-DIMENSIONAL STRETCHING FLOW OF AN OLDROYD FLUID*
}

\author{
BY \\ N. PHAN - THIEN \\ Sydney University, N.S.W., Australia
}

Summary. It is shown that a three-dimensional stretching flow of an Oldroyd-type fluid has an exact solution. As the fluid becomes Maxwellian, the solution permits a vortex sheet to propagate from the boundary into the flow domain. Furthermore, it is shown that there exists a critical Weissenberg number above which a stress component increases exponentially with time.

I. Introduction. In a recent paper Wang [1] showed that there are exact solutions to the flow of a Newtonian fluid which is caused by the stretching of a flat surface in two lateral directions. The flow geometry is depicted in Fig. 1, where the boundary conditions on the surface are

$$
u=a x, \quad v=b y, \quad w=0, \quad \text { at } z=0 .
$$

Here $\{u, v, w\}$ are the Cartesian components of the velocity vector and $a, b$ are some constants. The condition at $z=\infty$ is quiescent.

Although the flow is rather artificial and cannot be realized in the laboratory, it has an exact solution and thus will be valuable to numerical analysts. In some sense the flow is an analog of the stagnation flow which has been documented in detail in Schlichting [2]. The Newtonian stagnation flow kinematics have been shown to be valid for the Oldroyd- $B$ fluid [3] and it could be conjectured that the Newtonian stretching flow kinematics solve the corresonding Oldroyd- $B$ flow problem as well. This conjecture is shown to be true here for a class of Oldroyd fluids which includes the Oldroyd- $B$ fluid. Furthermore, it is shown that there exists a critical Weissenberg number above which one component of the stress tensor increases exponentially in time. This temporal instability is related to the unboundedness of the Trouton (or elongational) viscosity in elongational flows. It is also demonstrated numerically that the Maxwell-type constitutive equations allow for the propagation of a vortex sheet in this stretching flow.

*Received February 1, 1985. 
II. Analysis. We consider the flow of a viscoelastic fluid above a highly elastic membrane continuously stretched by uniform but increasing tension in directions $x$ and $y$ (Fig. 1). The velocities of a fluid particle on the membrane are given by (1). Wang [1] showed that the following flow kinematics solves the Newtonian problem:

$$
u=a x f^{\prime}(\xi), \quad v=a y g^{\prime}(\xi), \quad w=-\sqrt{(a v)}(f+g),
$$

where $\xi=z / \sqrt{(\nu / z)}$ is a normalized coordinate, $\nu$ is the kinematic viscosity of the Newtonian fluid, $f$ and $g$ are two functions of $\xi$ to be determined from the Navier-Stokes equations, and the prime denotes a derivative with respect to $\xi$. In this paper, we single out an Oldroyd-type constitutive equation which predicts constant viscosity, first and second normal stress coefficients in simple shearing flows and show that the kinematics (2) is also valid. The problem will be formulated as an initial-boundary-value problem so $f$ and $g$ are allowed to depend on the time $t$ as well.

Constitutive relation. We are concerned with an Oldroyd-type constitutive equation in which the "extra" stress is given by

$$
\tau=2 \eta_{s} \mathbf{D}+\mathbf{S},
$$

where $2 \eta_{s} \mathbf{D}$ is the solvent-contributed stress tensor and $\mathbf{S}$ obeys

$$
\mathbf{S}+\lambda\left\{\frac{\partial}{\partial t} \mathbf{S}+\mathbf{u} \cdot \nabla \mathbf{S}-\mathbf{L S}-\mathbf{S L}^{T}\right\}=2 \eta_{p}\left\{\mathbf{D}-\mu \lambda \mathbf{D}^{2}+\frac{1}{2} \mu \lambda \operatorname{tr}\left(\mathbf{D}^{2}\right) \mathbf{1}\right\} .
$$

In (3) and (4) $\mathbf{L}=(\nabla \mathbf{u})^{T}$ is the velocity gradient tensor, $\mathbf{D}$ is its symmetric part, $\mathbf{1}$ is the unit tensor, $\operatorname{tr}$ is the trace operation, the superscript $T$ denotes a transpose operation, and $\eta_{s}, \eta_{p}, \lambda$, and $\mu$ are some material constants. Note that (4) is a subclass of the Oldroyd- $B$-constant model [4]. When $\mu=0$, one has the Oldroyd- $B$ fluid, and if $\eta_{s}$ is also

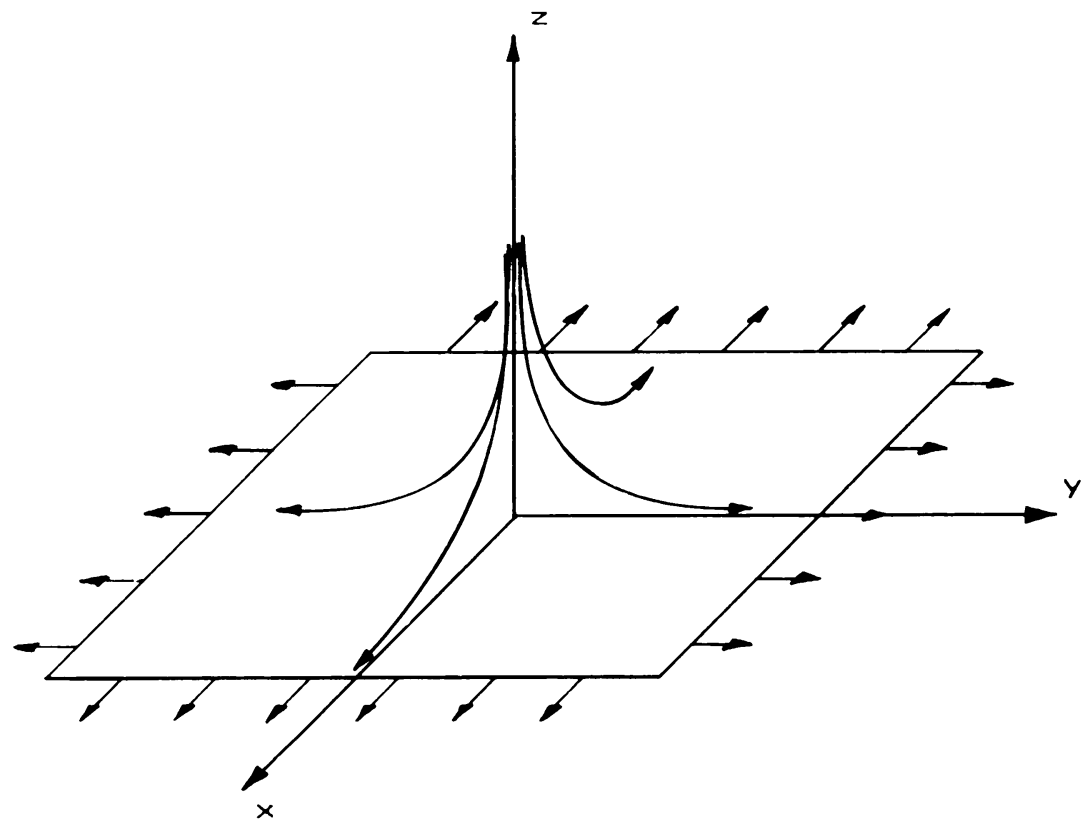

Fig. 1. The flow geometry. 
zero then one has the Maxwell fluid. In a simple shear flow with shear rate $\dot{\gamma},(3)$ and (4) predict that the fluid viscosity is

$$
\eta=\eta_{s}+\eta_{p}
$$

and the normal stress differences are

$$
\begin{gathered}
N_{1}=\tau_{x x}-\tau_{y y}=2 \eta_{p} \lambda \dot{\gamma}^{2}, \\
N_{2}=\tau_{y y}-\tau_{z z}=-\frac{1}{2} \mu \eta_{p} \lambda \dot{\gamma}^{2}=-\frac{1}{4} \mu N_{1} .
\end{gathered}
$$

Consequently, the normal stress ratio is $N_{2} / N_{1}=-\mu / 4$. From experimental evidence [5], $\mu$ is positive and of order unity.

In a uniaxial elongational flow, where

$$
[\mathbf{L}]=\operatorname{diag}\left(\gamma,-\frac{1}{2} \dot{\gamma},-\frac{1}{2} \dot{\gamma}\right),
$$

the elongational viscosity is given by

$$
\eta_{T}=\frac{N_{1}}{\dot{\gamma}}=3 \eta_{s}+3 \eta_{p} \frac{1-\frac{1}{2} \mu \lambda \dot{\gamma}(1-\lambda \dot{\gamma})}{(1-2 \lambda \dot{\gamma})(1+\lambda \dot{\gamma})},
$$

provided that $-1<\lambda \dot{\gamma}<1 / 2$. Outside this range there is no steady-state solution. The constitutive relation (4) with $\mu=0$ can be derived from a dilute suspension of dumbbell molecules. We expect that (4) will be a good model for the "Boger" fluids.

Governing equations. In the Cartesian coordinate system depicted in Fig. 1, the field equations are $\nabla \cdot \mathbf{u}=0=\rho d \mathbf{u} / d t-\nabla \cdot \boldsymbol{\sigma}$, where $d / d t$ is the material time derivative, $\rho$ is the fluid density, and $\boldsymbol{\sigma}=-P \mathbf{1}+\tau$ is the total stress tensor. Adopting the flow kinematics (2), with $\nu=\eta / \rho=\left(\eta_{p}+\eta_{s}\right) / \rho$, one finds that the stress components obey

$$
\begin{array}{r}
S_{x x}+\lambda\left\{\partial_{t} S_{x x}+a x f^{\prime} \partial_{x} S_{x x}+a y g^{\prime} \partial_{y} S_{x x}-a(f+g) S_{x x}^{\prime}-2 a\left(f^{\prime} S_{x x}+\frac{x}{l} f^{\prime \prime} S_{x z}\right)\right\} \\
=\eta_{p} a\left\{2 f^{\prime}-\mu \lambda a\left[f^{\prime 2}-g^{\prime 2}-\left(f^{\prime}+g^{\prime}\right)^{2}-\frac{1}{2} \frac{y^{2}}{l^{2}} g^{\prime \prime 2}\right]\right\}, \\
S_{y y}+\lambda\left\{\partial_{t} S_{y y}+a x f^{\prime} \partial_{x} S_{y y}+a y g^{\prime} \partial_{y} S_{y y}-a(f+g) S_{y y}^{\prime}-2 a\left[g^{\prime} S_{y y}+\frac{y}{l} g^{\prime \prime} S_{y z}\right]\right\} \\
=\eta_{p} a\left\{2 g^{\prime}-\mu \lambda a\left[g^{\prime 2}-f^{\prime 2}-\left(f^{\prime}+g^{\prime}\right)^{2}-\frac{1}{2} \frac{x^{2}}{l^{2}} f^{\prime \prime 2}\right]\right\} \\
S_{z z}+\lambda\left\{\partial_{t} S_{z z}+a x f^{\prime} \partial_{x} S_{z z}+a y g^{\prime} \partial_{y} S_{z z}-a(f+g) S_{z z}^{\prime}+2 a\left(f^{\prime}+g^{\prime}\right) S_{z z}\right\} \\
=-2 \eta_{p} a\left\{f^{\prime}+g^{\prime}+\mu \lambda a f^{\prime} g^{\prime}\right\} \\
S_{x y}+\lambda\left\{\partial_{t} S_{x y}+a x f^{\prime} \partial_{x} S_{x y}+a y g^{\prime} \partial_{y} S_{x y}-a(f+g) S_{x y}^{\prime}-a\left(f^{\prime}+g^{\prime}\right) S_{x y}\right. \\
\left.-a\left(\frac{x}{l} f^{\prime \prime} S_{y z}+\frac{y}{l} g^{\prime \prime} S_{x z}\right)\right\}=-\frac{1}{2}+\eta_{p} a^{2} \mu \lambda \frac{x y}{l^{2}} f^{\prime \prime} g^{\prime \prime}
\end{array}
$$




$$
\begin{array}{r}
S_{x y}+\lambda\left\{\partial_{t} S_{x z}+a x f^{\prime} \partial_{x} S_{x z}+a y g^{\prime} \partial_{y} S_{x z}-a(f+g) S_{x z}^{\prime}+a\left(f^{\prime}+g^{\prime}\right) S_{x z}-a f^{\prime} S_{x z}\right. \\
\left.-a \frac{x}{l} f^{\prime \prime} S_{z z}\right\}=\eta_{p} a \frac{x}{l}\left\{f^{\prime \prime}+\mu \lambda a g^{\prime} f^{\prime \prime}\right\}, \\
S_{y z}+\lambda\left\{\partial_{t} S_{y z}+a x f^{\prime} \partial_{x} S_{y z}+a y g^{\prime} \partial_{y} S_{y z}-a(f+g) S_{y z}^{\prime}+a f^{\prime} S_{y z}-a \frac{y}{l} g^{\prime \prime} S_{z z}\right\} \\
=\eta_{p} a \frac{y}{l}\left\{g^{\prime \prime}+\mu \lambda a f^{\prime} g^{\prime \prime}\right\},
\end{array}
$$

where $l=\sqrt{(\nu / a)}$ is the derived length scale (there is no natural time scale in this problem).

We define the Weissenberg number $\mathrm{Wi}$, a retardation parameter $\beta$, and the dimensionless time $\bar{t}$ by

$$
\mathrm{Wi}=\lambda a, \quad \beta=\eta_{p} / \eta, \quad \bar{t}=a t .
$$

Note that $\beta$ varies from 0 (Newtonian fluid) to 1 (Maxwell fluid). It is then found that the stress components are polynomials in $x$ and $y$. Explicitly,

$$
\begin{aligned}
& S_{x x}=\eta a\left\{\overline{X X}_{0}+\frac{x^{2}}{l^{2}} \overline{X X}_{1}+\frac{y^{2}}{l^{2}} \overline{X X}_{2}\right\}, \\
& S_{y y}=\eta a\left\{\overline{Y Y}_{0}+\frac{x^{2}}{l^{2}} \overline{Y Y}_{1}+\frac{y^{2}}{l^{2}} \overline{Y Y}_{2}\right\}, \\
& S_{z z}=\eta a \overline{Z Z}, \\
& S_{x y}=\eta a \frac{x y}{l^{2}} \overline{X Y}, \\
& S_{x z}=\eta a \frac{x}{y} \overline{X Z}, \\
& S_{y z}=\eta a \frac{y}{l} \overline{Y Z},
\end{aligned}
$$

where $\overline{X X}_{0}, \overline{X X}_{1}, \overline{X X}_{2}, \ldots, \overline{Y Z}$, ten in all, are functions of time $\bar{t}$ and $\xi=z / l$. They are governed by

$$
\begin{gathered}
\overline{X X}_{0}+\mathrm{Wi}\left\{\dot{X X}_{0}-(f+g) \overline{X X}_{0}^{\prime}-2 f^{\prime} \overline{X X}_{0}\right\}=2 \beta\left\{f^{\prime}+\mu \mathrm{Wi}\left(g^{\prime 2}+g^{\prime} f^{\prime}\right)\right\} \\
\overline{X X}_{1}+\mathrm{Wi}\left\{\dot{\overline{X X}}_{1}-(f+g) \overline{X X}_{1}^{\prime}-2 f^{\prime \prime} \overline{X Z}\right\}=0 \\
\overline{X X}_{2}+\mathrm{Wi}\left\{\dot{\overline{X X}}_{2}-(f+g) \overline{X X}_{2}^{\prime}+2 g^{\prime} \overline{X X}_{2}-2 f^{\prime} \overline{X X}_{2}\right\}=\frac{1}{2} \beta \mu \mathrm{Wi} g^{\prime \prime 2}, \\
\overline{Y Y}_{0}+\mathrm{Wi}\left\{\dot{\overline{Y Y}}_{0}-(f+g) \overline{Y Y}_{0}^{\prime}-2 g^{\prime} \overline{Y Y}_{0}\right\}=2 \beta\left\{g^{\prime}+\mu \mathrm{Wi}\left(f^{\prime 2}+f^{\prime} g^{\prime}\right)\right\} \\
\overline{Y Y}_{1}+\mathrm{Wi}\left\{\dot{\bar{Y}}_{1}-(f+g) \overline{Y Y}_{1}^{\prime}+2 f^{\prime} \overline{Y Y}_{1}-2 g^{\prime} \overline{Y Y}_{1}\right\}=\frac{1}{2} \beta \mu \mathrm{Wi} f^{\prime \prime 2}, \\
\overline{Y Y}_{2}+\mathrm{Wi}\left\{\dot{\overline{Y Y}}_{2}-(f+g) \overline{Y Y}_{2}^{\prime}-2 g^{\prime \prime} \overline{Y Z}\right\}=0 \\
\overline{X Y}+\mathrm{Wi}\left\{\dot{\bar{X}}_{X Y}-(f+g) \overline{X Y}^{\prime}-f^{\prime \prime} \overline{Y Z}-g^{\prime \prime} \overline{X Z}\right\}=-\frac{1}{2} \beta \mu \mathrm{Wi} f^{\prime \prime} g^{\prime \prime},
\end{gathered}
$$




$$
\begin{aligned}
& \overline{X Z}+\mathrm{Wi}\left\{\dot{\overline{X Z}}-(f+g) \overline{X Z}{ }^{\prime}+\left(f^{\prime}+g^{\prime}\right) \overline{X Z}-f^{\prime \prime} \overline{Z Z}\right\}=\beta\left(f^{\prime \prime}+\mu \mathrm{Wi} f^{\prime \prime} g^{\prime}\right), \\
& \overline{Y Z}+\mathrm{Wi}\left\{\dot{\overline{Y Z}}-(f+g) \overline{Y Z}{ }^{\prime}+\left(f^{\prime}+g^{\prime}\right) \overline{Y Z}-g^{\prime \prime} \overline{Z Z}\right\}=\beta\left(g^{\prime \prime}+\mu \mathrm{Wi} f^{\prime} g^{\prime \prime}\right), \\
& \overline{Z Z}+\mathrm{Wi}\left\{\dot{\overline{Z Z}}-(f+g) \overline{Z Z} \bar{Z}^{\prime}+2\left(f^{\prime}+g^{\prime}\right) \overline{Z Z}\right\}=-\beta\left\{f^{\prime}+g^{\prime}+\mu \mathrm{Wi} f^{\prime} g^{\prime}\right\}
\end{aligned}
$$

where the superdot denotes a time derivative with respect to time $\bar{t}$. Now to show that the chosen kinematics is exact, we must produce a scalar pressure to balance the equations of conservation of linear momentum. These equations are

$$
\begin{aligned}
& \frac{\partial P}{\partial x}=\eta a \frac{x}{l^{2}}\left\{(1-\beta) f^{\prime \prime \prime}+2 \overline{X X}_{1}+\overline{X Y}+\overline{X Z}-\left[\dot{f}^{\prime}+f^{\prime 2}-(f+g) f^{\prime \prime}\right]\right\} \\
& \frac{\partial P}{\partial y}=\eta a \frac{y}{l^{2}}\left\{(1-\beta) g^{\prime \prime \prime}+2 \overline{Y Y}_{2}+\overline{X Y}+\overline{Y Z}-\left[\dot{g}^{\prime}+g^{\prime 2}-(f+g) g^{\prime \prime}\right]\right\} \\
& \frac{\partial P}{\partial z}=\frac{\eta a}{l}\left\{-(1-\beta)\left(f^{\prime \prime}+g^{\prime \prime}\right)+\overline{X Z}+\overline{Y Z}+\overline{Z Z}+\left[\dot{f}+\dot{g}-(f+g)\left(f^{\prime}+g^{\prime}\right)\right]\right\}
\end{aligned}
$$

Note that the first term inside the curly brackets of (32)-(34) is the viscous contributed term, the next three are the non-Newtonian terms, and those inside the square brackets are inertia terms. Clearly, from (32)-(34),

$$
P=\frac{1}{2} \eta a\left\{C_{1} \frac{x^{2}}{l^{2}}+C_{2} \frac{y^{2}}{l^{2}}+2 Q\right\}
$$

where $C_{1}$ and $C_{2}$ are only functions of time and $Q$ is a function of $\xi$ and $\bar{t}$. These are given by

$$
\begin{aligned}
& C_{1}=(1-\beta) f^{\prime \prime \prime}+2 \overline{X X}_{1}+\overline{X Y}+\overline{X Z}-\dot{f}^{\prime}-f^{\prime 2}+(f+g) f^{\prime \prime}, \\
& C_{2}=(1-\beta) g^{\prime \prime \prime}+2 \overline{Y Y}_{2}+\overline{X Y}+\overline{Y Z}-\dot{g}^{\prime}-g^{\prime 2}+(f+g) g^{\prime \prime},
\end{aligned}
$$

and

$$
Q^{\prime}=-(1-\beta)\left(f^{\prime \prime}+g^{\prime \prime}\right)+\overline{X Z}+\overline{Y Z}+\overline{Z Z}+\dot{f}+\dot{g}-(f+g)\left(f^{\prime}+g^{\prime}\right) .
$$

From the quiescent conditions for the velocity and the stress field at $\xi=\infty$ we deduce that $C_{1}=C_{2} \equiv 0$. Thus, we have

$$
\begin{gathered}
(1-\beta) f^{\prime \prime \prime}+2 \overline{X X}_{1}+\overline{X Y}+\overline{X Z}-\dot{f}-f^{\prime 2}+(f+g) f^{\prime \prime}=0, \\
(1-\beta) g^{\prime \prime \prime}+2 \overline{Y Y}_{2}+\overline{X Y}+\overline{Y Z}-\dot{g}^{\prime}-g^{\prime 2}+(f+g) g^{\prime \prime}=0 .
\end{gathered}
$$

The proof is thus complete. Note that the time evolution of the velocity field is governed by (38), (39), (23), and (27)-(31). That is, $\overline{X X}_{0}, \overline{Y Y}_{0}, \overline{X X}_{2}$, and $\overline{Y Y}_{1}$ do not participate in determining $f$ and $g$. The governing equations are subject to the boundary conditions at

$$
\begin{gathered}
f+g=0, \quad f^{\prime}=1, \quad g^{\prime}=b / a \equiv \alpha \quad \text { at } \xi=0, \\
f^{\prime}=g^{\prime}=0 \quad \text { at } \xi=\infty .
\end{gathered}
$$


Without loss of generality we set $f=g=0$ at $\xi=0$ and restrict $\alpha$ to $[0,1]$; the case $\alpha>1$ can be included by simply interchanging the $x$ and $y$ axes (Wang [1]).

Initial solution. We assume that the flow is impulsively started from quiescent conditions. In a finite but small time $\bar{i}$ a boundary layer of thickness $O\left(\bar{t}^{1 / 2}\right)$ is developed near the membrane. An order of magnitude analysis on (39) and (40) based on this length scale leads to

$$
(1-\beta) f^{\prime \prime \prime}-\dot{f}, \quad 0<\bar{t} \lll 1,
$$

and an identical equation for $g$. With $0<t^{*}=(1-\beta) \lll 1$, this equation has the solution

$$
f(\xi, \bar{t})=\xi \operatorname{erfc}\left(\frac{\xi}{2 \sqrt{t^{*}}}\right)-2 \sqrt{\frac{t^{*}}{\pi}}\left[\exp \left(-\frac{\xi^{2}}{4 t^{* 2}}\right)-1\right],
$$

where $\operatorname{erfc}(\cdot)$ is the complementary error function. Similarly,

$$
g(\xi, \bar{t})=\alpha f(\xi, \bar{t}) .
$$

Note that the initial inflow at infinity is

$$
w(\infty, t)=2 a \sqrt{(\nu(1-\beta) t)}(1+\alpha), \quad 0<t \lll 1 .
$$

These initial solutions are used to start the numerical scheme described in Sec. III.

Limiting Weissenberg number. Since the flow at $z=0$ is biaxial flow, it is expected that there is a critical Weissenberg number above which a component of the stress tensor increases in time without bound. To show this we specialize (22), (25), and (31) to $\xi=0$ :

$$
\begin{gathered}
\overline{X X}_{0}+\mathrm{Wi}\left\{\dot{\overline{X X}}_{0}-2 \overline{X X}_{0}\right\}=2 \beta[1+\mu \alpha \mathrm{Wi}(1+\alpha)], \\
\overline{Y Y}_{0}+\mathrm{Wi}\left\{\dot{\overline{Y Y}}_{0}-2 \alpha \overline{Y Y}_{0}\right\}=2 \beta[\alpha+\mu \mathrm{Wi}(1+\alpha)], \\
\overline{Z Z}+\mathrm{Wi}\{\dot{\overline{Z Z}}+2(1+\alpha) \overline{Z Z}\}=-2 \beta[1+\alpha+\alpha \mu \mathrm{Wi}] .
\end{gathered}
$$

For quiescent conditions on the stress, these have solutions

$$
\begin{aligned}
& \overline{X X}_{0}(\bar{t})=\frac{2 \beta[1+\mu \mathrm{Wi} \alpha(1+\alpha)]}{1-2 \mathrm{Wi}}\left[1-\exp \left(-\frac{1-2 \mathrm{Wi}}{\mathrm{Wi}} \bar{t}\right)\right], \\
& \overline{Y Y}_{0}(\bar{t})=\frac{2 \beta[\alpha+\mu \mathrm{Wi}(1+\alpha)]}{1-2 \mathrm{Wi}}\left[1-\exp \left(-\frac{1-2 \alpha \mathrm{Wi}}{\mathrm{Wi}} \bar{t}\right)\right], \\
& \overline{Z Z}(\bar{t})=\frac{2 \beta(1+\alpha+\alpha \mu \mathrm{Wi})}{1+2(1+\alpha) \mathrm{Wi}}\left[1-\exp \left(-\frac{1+2(1+\alpha) \mathrm{Wi}}{\mathrm{Wi}} \bar{t}\right)\right] .
\end{aligned}
$$

Thus, if $\mathrm{Wi}<-\frac{1}{2}(1+\alpha)$ or $\mathrm{Wi}>\min \left(\frac{1}{2}, \frac{1}{2 \alpha}\right)$ at least one component of the stress increases exponentially with time. At these critical Weissenberg numbers, either $\overline{X X}_{0}$, $\overline{Y Y}_{0}$, or $\overline{Z Z}_{0}$ increases linearly with time. Since $\alpha \in[0,1]$, the upper Weissenberg number is $\frac{1}{2}$. However, because neither $\overline{X X}_{0}$ nor $\overline{Y Y}_{0}$ participates in determining the velocity field, a steady-state solution for $f$ and $g$ can be obtained even though $\mathrm{Wi} \geqslant \frac{1}{2}$. Here we are only concerned with $0<\mathrm{Wi} \leqslant \frac{1}{2}$. Although a steady-state velocity profile exists even when $\mathrm{Wi} \geqslant \frac{1}{2}$, a general-purpose computer program cannot be expected to find this steady-state solution simply because a steady-state stress field does not exist. 


\section{Numerical solution}

Numerical scheme. A closed-form steady-state solution can be obtained only for the Newtonian case $(\beta=0)$ and only when $\alpha=0$ where $g=0$ and [1]

$$
f=1-\exp (-\xi) \text {. }
$$

For $\alpha \neq 0$ and $\alpha \neq 1$ Wang [1] used a two-parameter minimization technique to obtain the steady-state Newtonian solution. Basically, initial guesses for $f^{\prime \prime}(0)$ and $g^{\prime \prime}(0)$ are supplied. The steady-state Newtonian equations (39) and (40) are then integrated from $\xi=0$ to $\xi=10$ (infinity) by a Runge-Kutta-Fehlberg method. The positive definite function

$$
J=\left|f^{\prime}\right|+\left|g^{\prime}\right|+\left|f^{\prime \prime}\right|+\left|g^{\prime \prime}\right| \text { at } \xi=10
$$

is evaluated. If it is not at minimum, new values of $f^{\prime \prime}(0)$ and $g^{\prime \prime}(0)$ are supplied by a Newton iteration method on $J=J\left(f^{\prime \prime}(0), g^{\prime \prime}(0)\right)$ and the process is repeated until convergence is obtained. The same method has been applied unsuccessfully to viscoelastic coaxial-disk flow [6] and stagnation problems [3] and will not be pursued further here. Instead, we use a simple time-marching scheme that has been applied successfully to several related viscoelastic problems (e.g., [3], [6]).

First, we rewrite (39) and (40) in the form

$$
\begin{aligned}
& \dot{f}=\dot{f}_{\infty}+(1-\beta) f^{\prime \prime}+\overline{X Z}-I, \\
& \dot{g}=\dot{g}_{\infty}+(1-\beta) g^{\prime \prime}+\overline{Y Z}-J,
\end{aligned}
$$

where $\dot{f}_{\infty}$ and $\dot{g}_{\infty}$ are the values of $\partial f / \partial \bar{t}$ and $\partial g / \partial \bar{t}$ at $\xi=\infty$, respectively, and $I, J$ are given by

$$
\begin{aligned}
& I=\int_{\xi}^{\infty}\left(2 \overline{X X}_{1}+\overline{X Y}-f^{\prime 2}+(f+g) f^{\prime \prime}\right) d \xi^{\prime}, \\
& J=\int_{\xi}^{\infty}\left(2 \overline{Y Y}_{2}+\overline{X Y}-g^{\prime 2}+(f+g) g^{\prime \prime}\right) d \xi^{\prime} .
\end{aligned}
$$

Note that

$$
\begin{aligned}
& \dot{f}_{\infty}=\left(I-\overline{X Z}-(1-\beta) f^{\prime \prime}\right) \quad \text { at } \xi=0, \\
& \dot{g}_{\infty}=\left(J-\overline{Y Z}-(1-\beta) g^{\prime \prime}\right) \quad \text { at } \xi=0 .
\end{aligned}
$$

Next, the interval $\xi \in[0, L]$ is divided into $N$ subintervals of equal length $\Delta \xi=L / N$. Based on the Newtonian solution, $L$ is taken to be of order 10 . The nodal value of the field variable $\psi(\xi, t)$ (which can be $f, g, \overline{X X}_{1}, \overline{Y Y}_{2}, \overline{X Y}, \overline{X Z}, \overline{Y Z}$, or $\overline{Z Z}$ ) at $\xi_{i}=(i-1) \Delta \xi$, $i=1, \ldots, N+1$, and at time $\bar{t}_{n}=n \Delta \bar{t}$ is denoted by $\psi_{i}^{n}$. The governing equations (53), (54), (23), and (27)-(31) are discretized by using a second-order finite-difference scheme for the spatial derivatives and first-order finite difference for the time derivatives:

$$
\psi_{i}^{\prime n}=\frac{\pi_{i+1}^{n}-\psi_{i-1}^{n}}{2 \Delta \xi}, \quad \psi_{i}^{\prime \prime n}=\frac{\psi_{i+1}^{n}-2 \psi_{i}^{n}+\psi_{i-1}^{n}}{\Delta \xi^{2}}, \quad \dot{\psi}_{i}^{n}=\frac{\psi_{i}^{n+1}-\psi_{i}^{n}}{\Delta \bar{t}} .
$$

Finally, one obtains a finite-difference system

$$
\psi^{n+1}=\psi^{n}+\Delta \bar{t} F\left(\psi^{n}\right)
$$


where $\psi$ is the solution vector (the nodal values of $f, g, \overline{X X}_{1}, \overline{Y Y}_{2}, \overline{X Y}, \overline{X Z}, \overline{Y Z}$, and $\overline{Z Z}$ ). Boundary conditions on $f$ and $g$ are satisfied by inventing fictitious nodes for $f$ and $g$ at $\xi_{0}=-\Delta \xi$ and $\xi_{n+2}=(N+1) \Delta \xi$ such that

$$
f_{0}=f_{2}-2 \Delta \xi, \quad g_{0}=g_{2}-2 \alpha \Delta \xi, \quad f_{N+2}=f_{N}, \quad g_{N+2}=g_{N} .
$$

This finite-difference scheme is started using the initial solution (43) and (44) where $\bar{t}=0.0001$. In sum, the basic numerical steps are:

(a) Using the current solution, evaluate $I, J, \dot{f}_{\infty}$, and $\dot{g}_{\infty}$ using (54)-(57). Integrals are computed by Simpson's rule.

(b) Evaluate $F\left(\psi^{\prime \prime}\right)$ using the current solution and the information obtained in step (a).

(c) Update the solution vector using (59). The process is repeated until steady state $\left(f^{\prime \prime}(0)\right.$ and $g^{\prime \prime}(0)$ are unchanged to three decimal places) is achieved. All computing was done on a VAX/780 in single precision mode with $\Delta \xi=0.05-0.1$ and $\Delta \bar{t}=0.0005-0.001$.

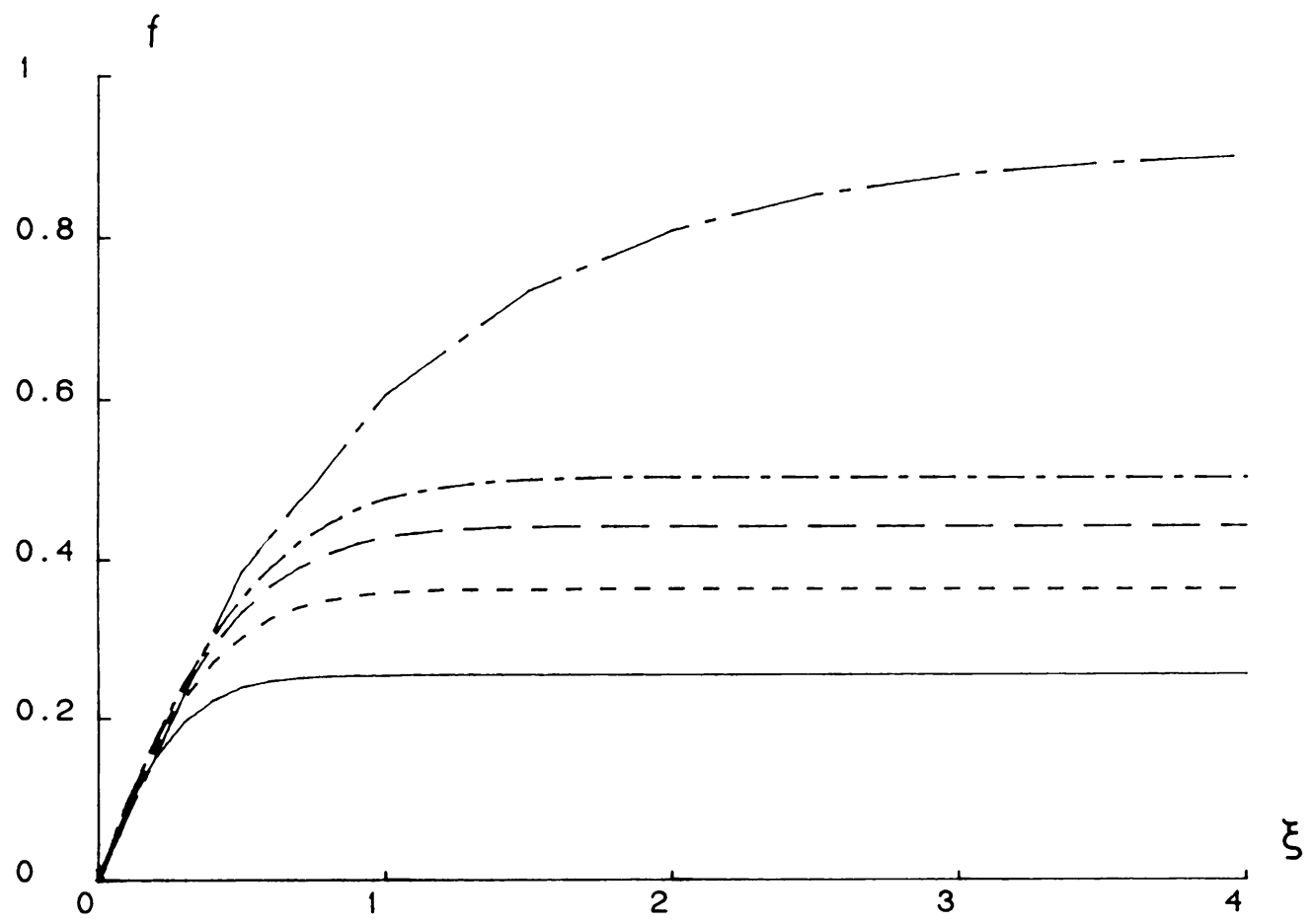

FIG. 2(a). Time evolution of $f$ for the case $\alpha=0, \mathrm{Wi}=0.5, \beta=0.5, \mu=0$. In this case $g \equiv 0$. The steady-state $f$ is also plotted.

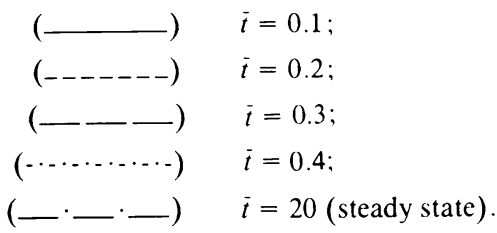




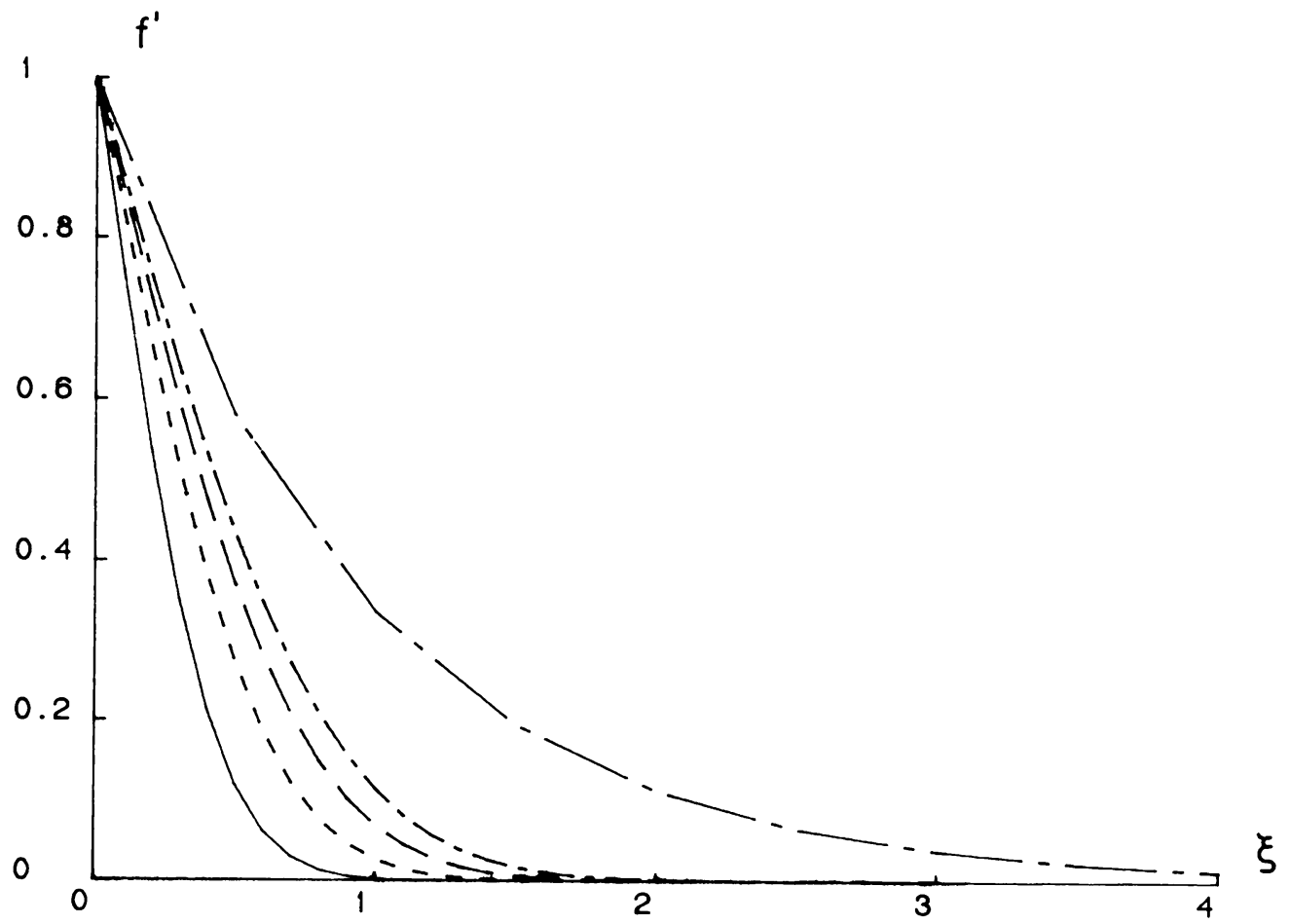

FIG. 2(b). Time evolution of $f^{\prime}$. Parameter values as in (2a).

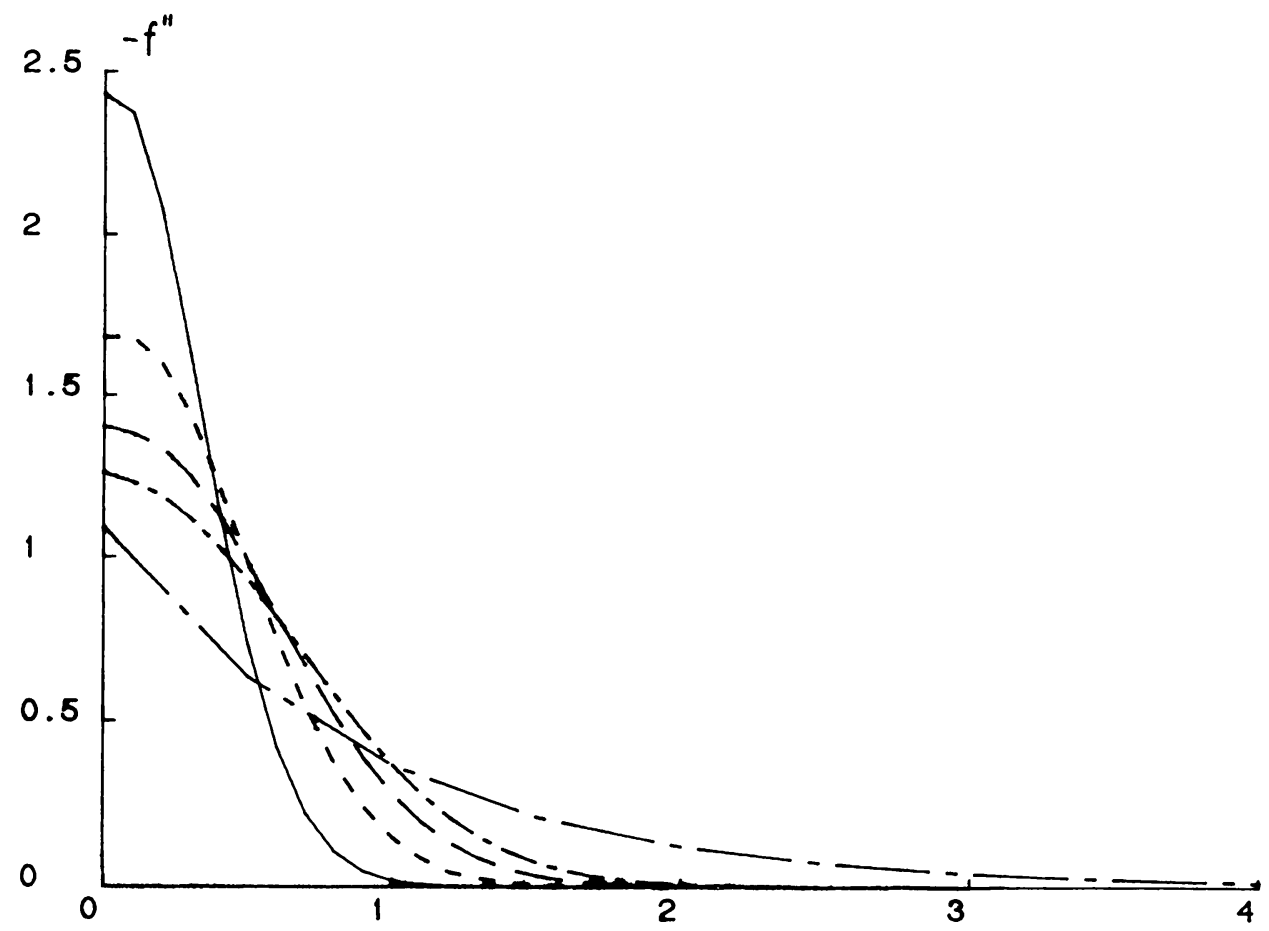

FIG. 2(c). Time evolution of $f^{\prime \prime}$. Parameter values as in (2a). 
Numerical results. To judge the accuracy of the finite-difference scheme we compare our steady-state numerical results for the Newtonian case $(\beta=0)$ with those of Wang [1]. In all cases, ranging from $\alpha=0$ to $\alpha=1$ we find agreement with Wang's results to three decimal places. This is deemed to be a success, considering the simple nature of the numerical scheme and the coarse mesh used $(\Delta \xi=0.1)$

In Fig. 2a-c we plot the time evolution of $f, f^{\prime}$, and $f^{\prime \prime}$ for the case $\alpha=0, \mathrm{Wi}=0.5$, $\beta=0.5$, and $\mu=0$. In this case, $g=0$ and one finds a Newtonian-like response where the vorticity (which is proportional to $f^{\prime \prime}$ ) is seen diffusing away from the boundary.

As $\beta$ is increased toward 1 (Maxwell fluid) the flow changes in character and indicates that there is a propagation of a vortex sheet, i.e., a discontinuity in $f^{\prime}$. Unfortunately, our numerical scheme cannot be guaranteed to capture true transience behavior when $\beta=1$; large "spurious" oscillations were seen. These oscillations seem to depend on the mesh size used and must be numerical noises which propagate in the fluid. The oscillations do not persist, and good steady-state solutions can still be obtained by the present method when $\beta=1$. The presence of the vortex sheet can be deduced from Fig. 3a-c, where the time

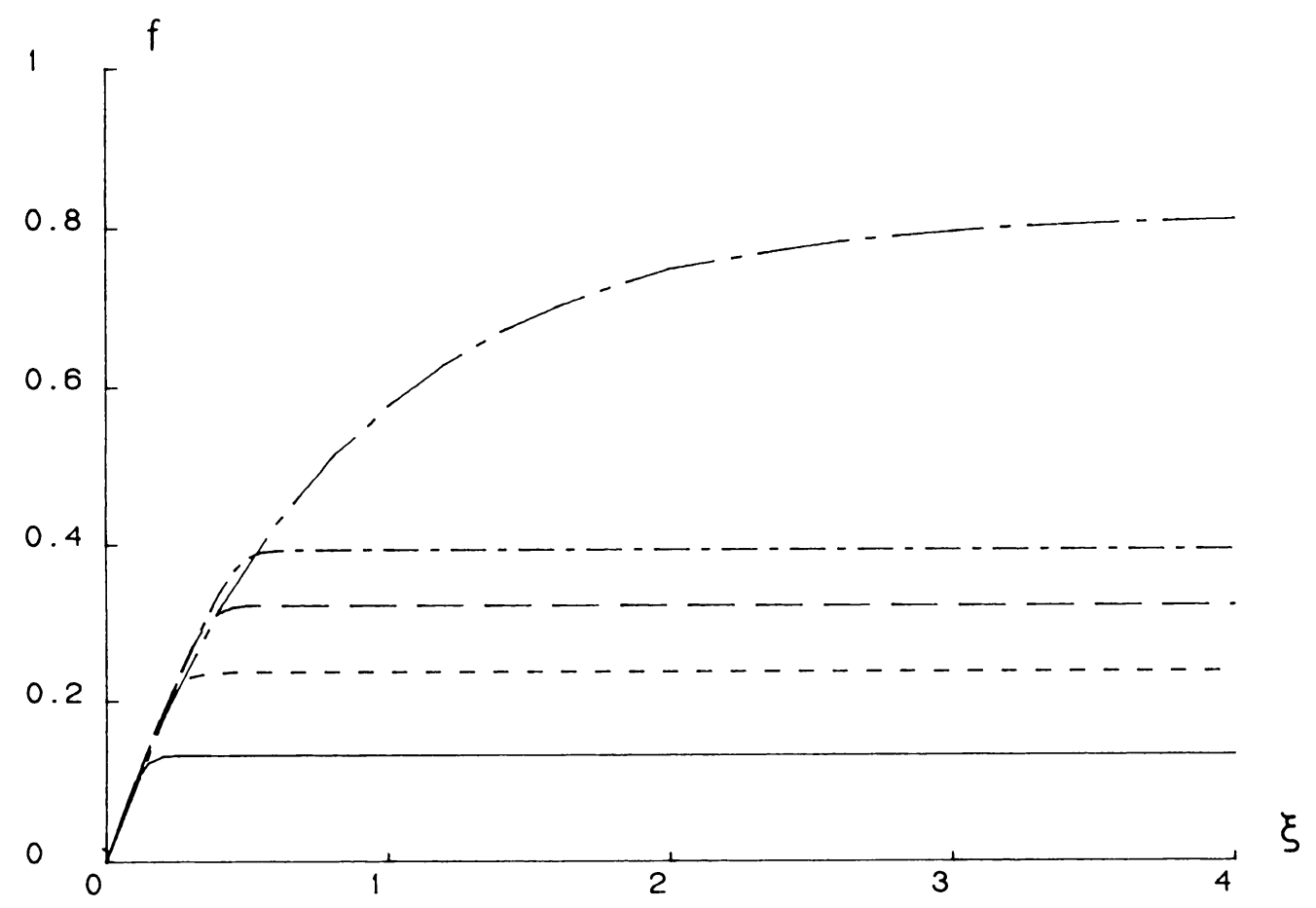

FIG. 3(a). Time evolution of $f$ for the case $\alpha=0$, Wi $=0.5, \beta=0.99$, $\mu=0$. The steady-state $f$ is also plotted.

$$
\begin{aligned}
& \text { (—) } \quad \bar{t}=0.1 \text {; } \\
& \text { (------) } \quad \bar{t}=0.2 \text {; } \\
& \text { (———) } \quad \bar{i}=0.3 \text {; } \\
& (\cdots \cdots \cdots) \quad \bar{i}=0.4 \text {; } \\
& \text { (_- - - } \quad \bar{t} \quad \bar{t}=20 \text { (steady state) }
\end{aligned}
$$




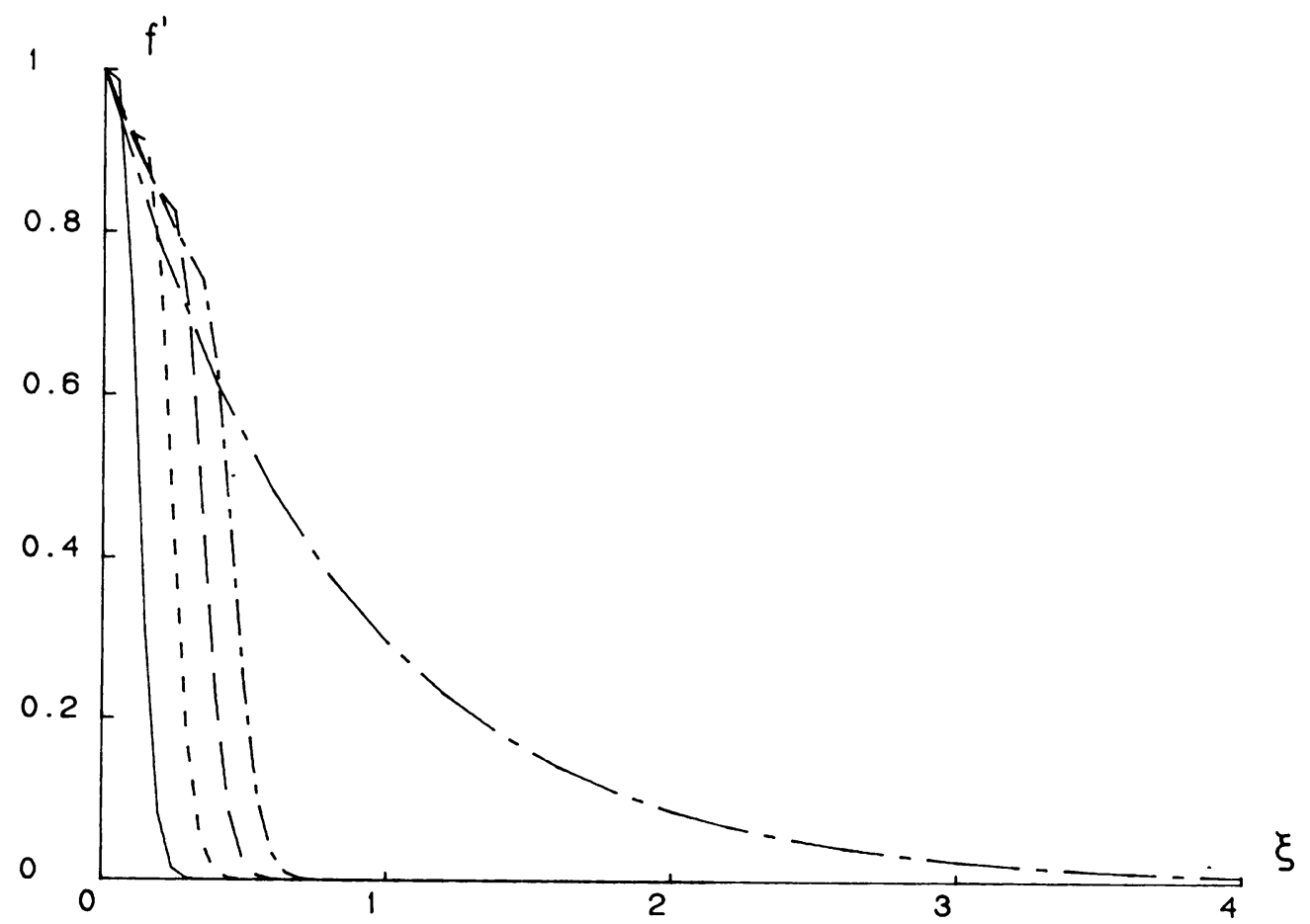

FIG. 3(b). Time evolution of $f^{\prime}$. Parameter values as in (3a).

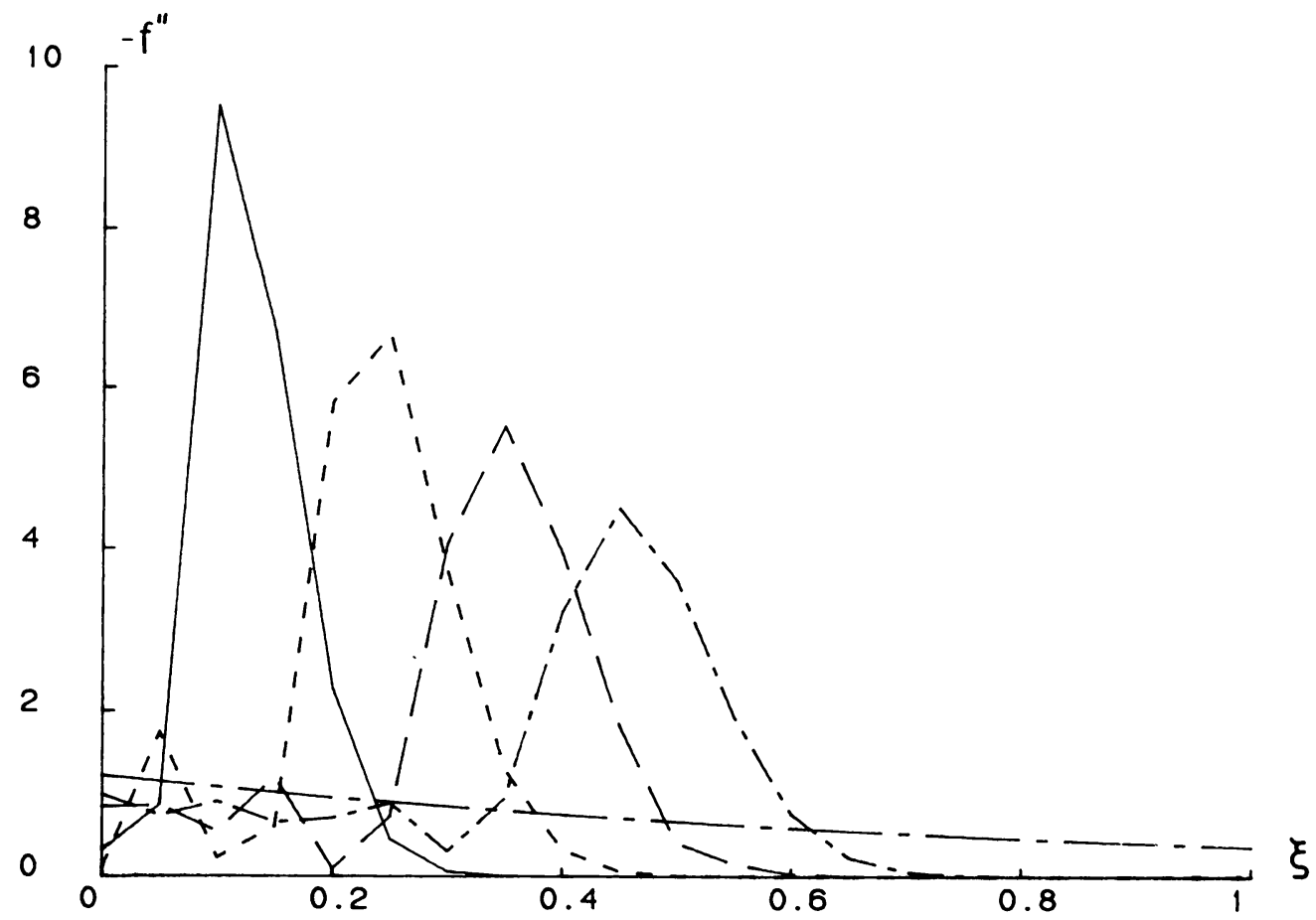

FIG. 3(c). Time evolution of $f^{\prime \prime}$. Parameter values as in (3a). 
TABLE 1. Values of $f(\infty), f^{\prime \prime}(0)$ at $\alpha=0$ and $\beta=0.5$ in the case $g \equiv 0$. The Newtonian values are $(1,-1)$.

\begin{tabular}{|c|c|c|c|c|c|}
\hline$u^{\mathrm{Wi}}$ & 0.1 & 0.2 & 0.3 & 0.4 & 0.5 \\
\hline 0 & $0.908, \quad-1.02$ & $0.959,-1.04$ & $0.945, \quad-1.06$ & $0.930,-1.08$ & $0.916, \quad-1.09$ \\
\hline 1 & $0.980,-1.02$ & $0.959,-1.04$ & $0.945,-1.06$ & $0.930,-1.08$ & $0.916,-1.09$ \\
\hline 2 & $0.900, \quad-1.02$ & $0.959, \quad-1.04$ & $0.945,-1.06$ & $0.930, \quad-1.08$ & $0.916, \quad-1.09$ \\
\hline
\end{tabular}

TABI.E 2. Values of $f(\infty), f^{\prime \prime}(0)$ at $\alpha=1$ and $\beta=0.5$ in the case $g \equiv f$. The

Newtonian values are $(0.752,-1.17)$.

\begin{tabular}{|c|c|c|c|c|c|}
\hline$\mu^{\mathrm{Wi}}$ & 0.1 & 0.2 & 0.3 & 0.4 & 0.5 \\
\hline 0 & $0.714, \quad-1.24$ & $0.698, \quad-1.29$ & $0.669, \quad-1.33$ & $0.654, \quad-1.36$ & $0.664, \quad-1.38$ \\
\hline 1 & $0.719, \quad-1.21$ & $0.697, \quad-1.24$ & $0.681,-1.25$ & $0.668,-1.26$ & $0.659, \quad-1.26$ \\
\hline 2 & $0.725, \quad-1.19$ & $0.706, \quad-1.19$ & $0.693,-1.19$ & $0.682,-1.18$ & $0.674, \quad-1.17$ \\
\hline
\end{tabular}

TABle 3. Values of $f(\infty), g(\infty)$ (first row) and $f^{\prime \prime}(0), g^{\prime \prime}(0)$ (second row) at $\alpha=0.5$ and $\beta=0.5$. The Newtonian values are $(f(\infty), g(\infty))=$ $(0.842,0.452)$ and $\left(f^{\prime \prime}(0), g^{\prime \prime}(0)\right)=(-1.09,-0.465)$.

\begin{tabular}{|c|c|c|c|c|c|}
\hline$\mu^{\mathrm{Wi}}$ & 0.1 & 0.2 & 0.3 & 0.4 & 0.5 \\
\hline 0 & $\begin{array}{cr}0.811, & 0.434 \\
-1.14, & -0.488\end{array}$ & $\begin{array}{rr}0.787, & 0.421 \\
-1.18, & -0.506\end{array}$ & \begin{tabular}{|cc}
0.768, & 0.411 \\
-1.21, & -0.52
\end{tabular} & $\begin{array}{rr}0.753, & 0.403 \\
-1.23, & -0.530\end{array}$ & $\begin{array}{rr}0.741, & 0.397 \\
-1.25, & -0.539\end{array}$ \\
\hline 1 & $\begin{array}{rr}0.813, & 0.438 \\
-1.13, & -0.477\end{array}$ & $\begin{array}{rr}0.792, & 0.429 \\
-1.15, & -0.483\end{array}$ & $\begin{array}{rr}0.774, & 0.420 \\
-1.17, & -0.486\end{array}$ & $\begin{array}{rr}0.760, & 0.414 \\
-1.18, & -0.487\end{array}$ & $\begin{array}{rr}0.749, & 0.410 \\
-1.20, & -0.487\end{array}$ \\
\hline 2 & $\begin{array}{rr}0.816, & 0.442 \\
-1.11, & -0.467\end{array}$ & $\begin{array}{rr}0.796, & 0.434 \\
-1.13, & -0.464\end{array}$ & $\begin{array}{rr}0.781, & 0.429 \\
-1.14, & -0.458\end{array}$ & $\begin{array}{cr}0.767, & 0.424 \\
-1.14, & -0.453\end{array}$ & $\begin{array}{rr}0.757, & 0.421 \\
-1.15, & -0.447\end{array}$ \\
\hline
\end{tabular}

TABLE 4. Same as in Table 3 except that $\beta=1$.

\begin{tabular}{|r|rr|rr|rr|cr|cr|}
\hline \multicolumn{2}{|c|}{ Wi } & \multicolumn{2}{|c|}{0.1} & \multicolumn{2}{c|}{0.2} & \multicolumn{2}{c|}{0.3} & \multicolumn{2}{|c|}{0.4} & \multicolumn{2}{c|}{0.5} \\
\hline & 0.778, & 0.416 & 0.727, & 0.387 & 0.685, & 0.364 & 0.650, & 0.345 & 0.620, & 0.328 \\
\hline 0 & -1.19, & -0.517 & -1.28, & -0.564 & -1.37, & -0.606 & -1.45, & -0.646 & -1.52, & -0.683 \\
\hline & 0.783, & 0.424 & 0.737, & 0.403 & 0.698, & 0.387 & 0.665, & 0.374 & 0.637, & 0.363 \\
1 & -1.16, & -0.491 & -1.22, & 0.503 & -1.26, & -0.507 & -1.30, & -0.504 & -1.32, & -0.498 \\
\hline & 0.789, & 0.431 & 0.747, & 0.417 & 0.712, & 0.405 & 0.684, & 0.395 & 0.659, & 0.387 \\
2 & -1.14, & -0.468 & -1.16, & -0.460 & -1.18, & -0.446 & -1.18, & -0.430 & -1.18, & -0.415 \\
\hline
\end{tabular}


responses of $f, f^{\prime}$, and $f^{\prime \prime}$ are plotted for the case $\alpha=0, \mathrm{Wi}=0.5, \beta=0.99$, and $\mu=0$ (a nearly Maxwell fluid). From Fig. $3 \mathrm{c}$ the vorticity $\left(f^{\prime \prime}\right)$ is seen to propagate from the boundary while being smoothed out by a small viscous effect (of viscosity $1-\beta$ ). The speed of this propagation is estimated to be of $O\left(\mathrm{Wi}^{1 / 2}\right)$.

Steady-state profiles for $f$ are also included in Figs. 2 and 3; they are nearly exponential in character and do not differ much from the Newtonian solution (52).

To help gauge the effects of various parameters in the problem, Tables 1-4 show the steady-state values of $f(\infty), g(\infty), f^{\prime \prime}(0)$, and $g^{\prime \prime}(0)$. From these tables it is clear that the inflow from infinity $(\sim f(\infty)+g(\infty))$ decreases as the Weissenberg number increases; it also decreases as the normal stress ratio $N_{2} / N_{1}=-\mu / 4$ increases. The values of $f^{\prime \prime}(0)$ and $g^{\prime \prime}(0)$ can be used to construct steady-state solutions by integrating the governing equations in $\xi$ as done by Wang [1]; however, we shall not be concerned with this.

Finally, the steady-state velocity and stress profiles are given in Fig. 4 for the case where $\alpha=0.5, \mathrm{Wi}=0.5, \mu=1$, and $\beta=1$. The largest component of the stress is $\overline{Z Z}$.

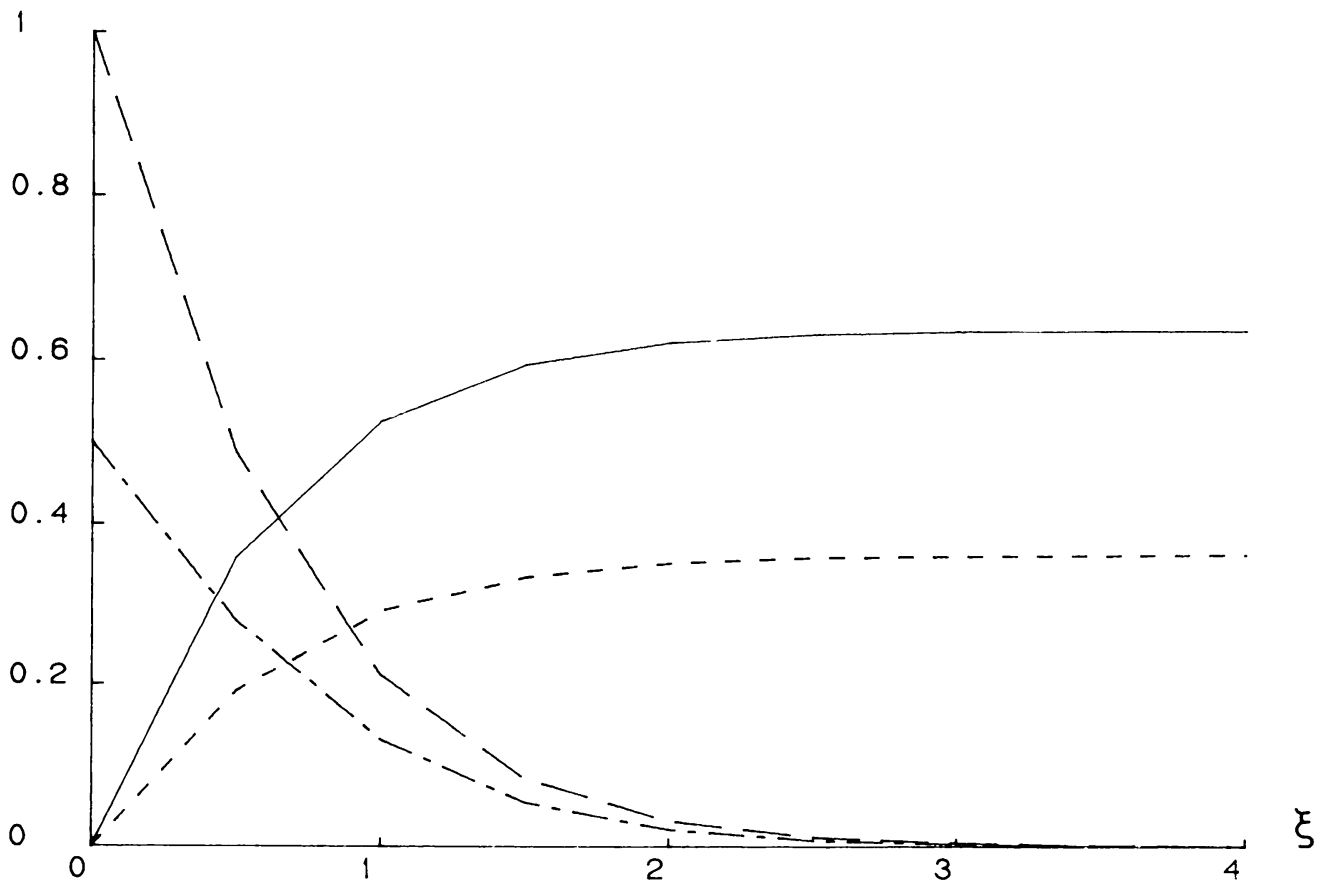

FIG. 4(a). Steady-state velocity field for the case $\alpha=0.5, \mathrm{Wi}=0.5, \beta=1, \mu=1$.

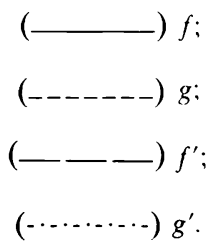




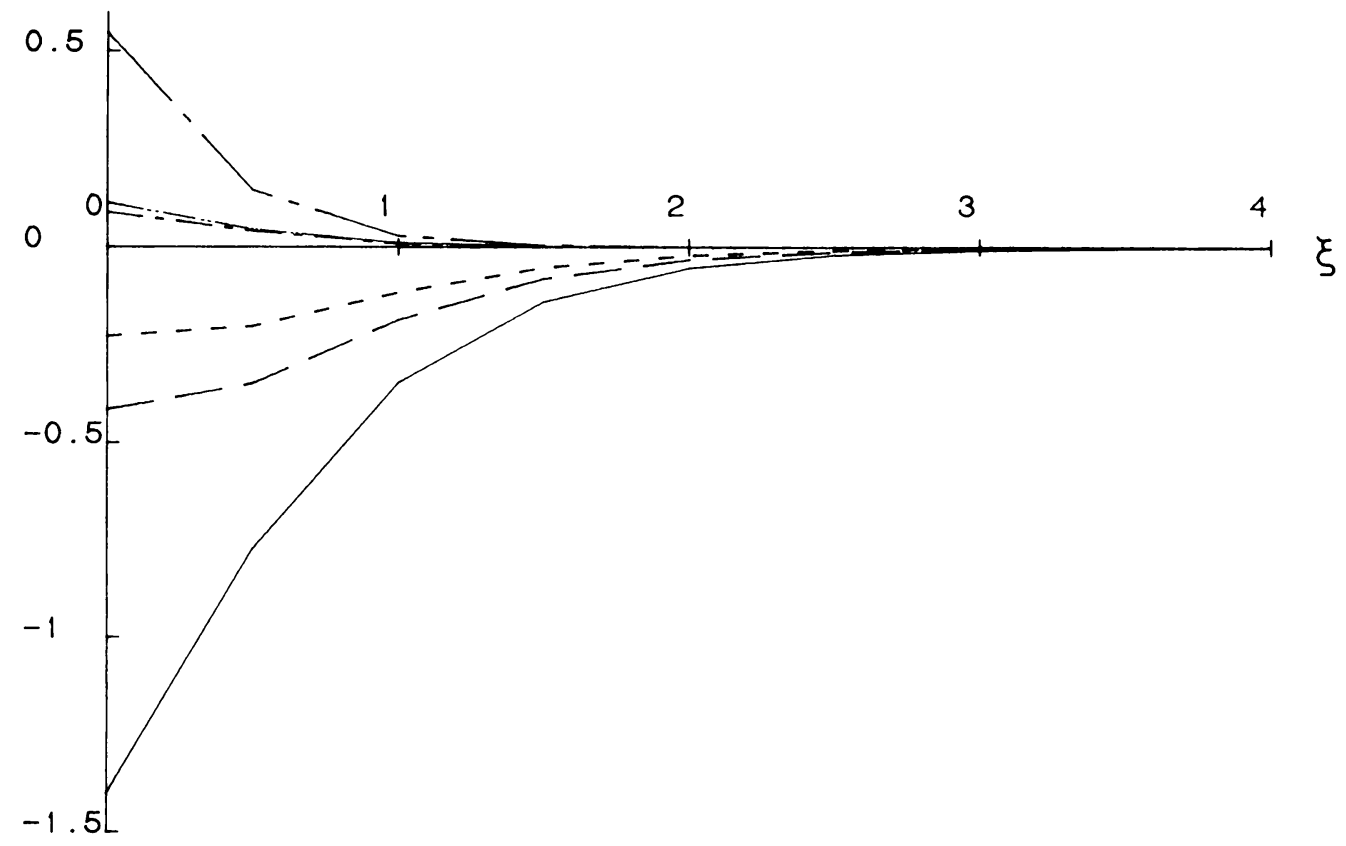

Fig. 4(b). Steady-state components of the stresses. Parameter values as in (4a).

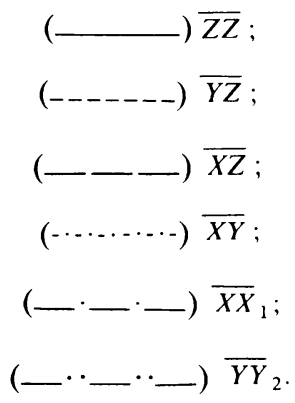

IV. Conclusions. To sum up, we have shown that the three-dimensional stretching flow of an Oldroyd-type fluid has an exact kinematics which has the same functional form as the Newtonian one. Furthermore, if the Weissenberg number exceeds $\frac{1}{2}$ there is no steady-state solution for the stress field, although a steady-state solution for the velocity field can be obtained. Numerical solutions obtained with a time-marching process reveal the difference between the Maxwell-type constitutive law $(\beta=1)$ and the Oldroyd- $B$-type constitutive law $(\beta<1)$. In the former, a vortex sheet is seen propagating from the boundary into the flow domain. In the latter, the vorticity diffuses from the boundary into the flow domain in a manner similar to that of a Newtonian fluid.

Acknowledgment. This research is supported by an ARGS (Australian Research Grant Scheme) project grant. The support is gratefully acknowledged. 


\section{REFERENCES}

[1] C. Y. Wang, The three-dimensional flow due to a stretching flat surface, Phys. Fluids 27, 1915-1917 (1984)

[2] H. Schlichting, Boundary layer theory, trans. J. Kestin, 6th ed., McGraw-Hill, New York, 1968

[3] N. Phan-Thien, Stagnation flows for the Oldroyd-B fluid, Rheol. Acta 23, 172-176 (1984)

[4] J. G. Oldroyd, Non-Newtonian effects in steady motion of some idealized elastico-viscous liquids, Proc. Roy. Soc. London Ser. A 245, 278-297 (1958)

[5] R. I. Tanner, Engineering rheology, Oxford University Press, London, 1985

[6] N. Phan-Thien, Coaxial-disk flow of an Oldroyd-B fluid: exact solution and stability, J. Non-Newtonian Fluid Mech. 13, 325-340 (1983) 